\title{
Three-Dimensional Classical Groups Acting on Polytopes
}

\author{
Peter A. Brooksbank • Deborah A. Vicinsky
}

Received: 3 April 2009 / Revised: 27 June 2009 / Accepted: 28 June 2009 /

Published online: 14 July 2009

(C) Springer Science+Business Media, LLC 2009

\begin{abstract}
Let $V$ be a three-dimensional vector space over a finite field. We show that any irreducible subgroup of GL $(V)$ that arises as the automorphism group of an abstract regular polytope preserves a nondegenerate symmetric bilinear form on $V$. In particular, the only classical groups on $V$ that arise as automorphisms of such polytopes are the orthogonal groups.
\end{abstract}

Keywords Abstract regular polytope $\cdot$ String C-group · Classical group

\section{Introduction}

This paper is a contribution to the recent effort to determine which members of a specific infinite family of finite simple groups can arise as the group of automorphisms of an abstract regular polytope (which we shall henceforth refer to simply as regular polytope). The combined results of Sjerve and Cherkassoff, and Leemans and Schulte, for example, determine all of the finite fields $\mathbb{F}_{q}$ for which $\operatorname{PSL}\left(2, \mathbb{F}_{q}\right)$ acts on a regular polytope. Specifically, $\operatorname{PSL}\left(2, \mathbb{F}_{q}\right)$ is the group of automorphisms of a regular polytope of rank 3 if and only if $q \notin\{2,3,7,9\}[5]$, whereas only $\operatorname{PSL}\left(2, \mathbb{F}_{11}\right)$ and $\operatorname{PSL}\left(2, \mathbb{F}_{19}\right)$ act on regular polytopes of rank 4 [2].

In this paper we examine the actions on regular polytopes of classical groups defined on a three-dimensional vector space $V$ over a finite field $\mathbb{F}_{q}$. Following the notation of [1, Sect. 2.1], $\kappa$ denotes a bilinear, hermitian or quadratic form on $V$, $I=I(V, \kappa)$ is the group of $\kappa$-isometries of $V$, and $\Omega=\Omega(V, \kappa)=I^{\prime}$, the derived subgroup of $I$. Note that if $\kappa$ is identically 0 , then $I(V, \kappa)=\operatorname{GL}(V)$; otherwise $\kappa$ is

P.A. Brooksbank $(\varangle) \cdot$ D.A. Vicinsky

Department of Mathematics, Bucknell University, Lewisburg, PA 17837, USA

e-mail: pbrooksb@bucknell.edu

D.A. Vicinsky

e-mail: dav005@bucknell.edu 
assumed to be nondegenerate. We define a classical group on $V$ to be any group $G$ satisfying $\Omega(V, \kappa) \leq G \leq I(V, \kappa)$ for some suitable $\kappa$.

In view of $[2,5]$, it seems reasonable to hope that one might find new examples of regular polytopes $\mathcal{P}$ for which $\Gamma(\mathcal{P})$, the group of automorphisms of $\mathcal{P}$, is isomorphic to a classical subgroup of $\operatorname{GL}(V)$. The main result of this paper demonstrates, however, that this is not the case.

Theorem If $\mathcal{P}$ is a regular polytope such that $\Gamma(\mathcal{P})$ is isomorphic to $G \leq \mathrm{GL}\left(3, \mathbb{F}_{q}\right)$ acting absolutely irreducibly on $V=\mathbb{F}_{q}^{3}$, then $q$ is odd, and there is a nondegenerate, symmetric bilinear form $\mathbf{f}$ on $V$ such that $\Omega(V, \mathbf{f}) \leq G \leq I(V, \mathbf{f})$.

Since $\Omega(V, \mathbf{f}) \cong \operatorname{PSL}\left(2, \mathbb{F}_{q}\right)$ for any nondegenerate, symmetric bilinear form $\mathbf{f}$ on $V=\mathbb{F}_{q}^{3}$, the fields $\mathbb{F}_{q}$ that admit flag-transitive actions of three-dimensional classical groups on regular polytopes are determined in $[2,5]$.

Related to our main theorem is (one aspect of) an investigation of B. Monson and E. Schulte into modular reduction of crystallographic Coxeter groups. In [4, Sect. 5] they describe explicitly the modular polytopes of rank 3 and construct several interesting families of such polytopes whose automorphism groups are isomorphic to groups $G$ satisfying $\Omega(V, \mathbf{f}) \leq G \leq I(V, \mathbf{f})$, where $V=\mathbb{F}_{p}^{3}$ (defined over a prime field), and $\mathbf{f}$ is a symmetric bilinear form on $V$.

As in [2], we find it convenient to employ a purely group-theoretic description of a group $G$ acting on a regular polytope. A group $G$ is a $C$-group if it has a generating sequence of involutions $t_{0}, t_{1}, \ldots, t_{r-1}$ satisfying the following intersection property:

$$
\forall I, J \subseteq\{0, \ldots, r-1\}: \quad\left\langle t_{i}: i \in I\right\rangle \cap\left\langle t_{j}: j \in J\right\rangle=\left\langle t_{k}: k \in I \cap J\right\rangle .
$$

A $C$-group $G$ is a string $C$-group if the following condition also holds:

$$
\forall i, j \in\{0, \ldots, r-1\}: \quad\left[t_{i}, t_{j}\right]=1 \text { if and only if }|i-j|>1 .
$$

The integer $r$ is the rank of $G$, and we write $G=\left\langle t_{0}, \ldots, t_{r-1}\right\rangle$ to emphasize a specific generating sequence of involutions satisfying (1) and (2). It is well known that, to each string $C$-group $G$ of rank $r$, one can associate an explicit regular polytope $\mathcal{P}$ of rank $r$ such that $G$ is isomorphic to $\Gamma(\mathcal{P})$; the reader is referred to [3, Theorem 2E11] for details of its construction.

The techniques that we utilize to prove our main result are quite different from the approach taken in [2]. The latter makes use of a very detailed understanding of the subgroup structure of $\operatorname{PSL}\left(2, \mathbb{F}_{q}\right)$. Our approach is to characterize interactions between involutions in $\operatorname{GL}\left(3, \mathbb{F}_{q}\right)$ in terms of the geometry of the projective plane. We show that geometric constraints arising from commutativity relations among sequences of involutions in $\operatorname{GL}\left(3, \mathbb{F}_{q}\right)$ satisfying (1) and (2) impose substantial restrictions on the groups they can generate.

\section{The Geometry of $\operatorname{GL}\left(3, \mathbb{F}_{q}\right)$}

In this section we collect the geometric properties of involutions in $\operatorname{GL}\left(3, \mathbb{F}_{q}\right)$ that we will need in the proofs of our main results. 
Let $V=\mathbb{F}_{q}^{3}$ be the natural vector space underlying $\operatorname{GL}\left(3, \mathbb{F}_{q}\right)$. We shall work with the projective plane, $\mathbb{P}(V)$, using the terms point for 1-space and line for 2-space. Accordingly we often compute with subgroups of GL( $V)$ via their projective action on $\mathbb{P}(V)$; an element or subgroup of $\mathrm{GL}(V)$ fixes a point or line in this action if it stabilizes the associated subspace of $V$.

If $\Delta$ is a line in $\mathbb{P}(V)$ and $\delta$ is a point on $\Delta$, then $T_{\delta \Delta}$ denotes the transvection group containing those elements of GL( $V)$ that act as the identity on $\Delta$ and on $V / \delta$. One says that $\delta$ (respectively $\Delta$ ) is the center (respectively axis) of the transvection group. Observe that $T_{\delta \Delta}$ fixes precisely the points on $\Delta$ and the lines through $\delta$, and is isomorphic to the additive group $\mathbb{F}_{q}$. If $T_{\gamma \Gamma}$ is another transvection group, $1 \neq s \in T_{\delta \Delta}$, and $1 \neq t \in T_{\gamma \Gamma}$, then we have the following elementary observation:

$$
[s, t]=1 \quad \text { if and only if } \delta=\gamma \text { or } \Delta=\Gamma .
$$

If $\operatorname{char}\left(\mathbb{F}_{q}\right)=2$, all involutions of $\operatorname{GL}(V)$ are transvections, and they are conjugate.

If $\operatorname{char}\left(\mathbb{F}_{q}\right)>2$, then an involution $t$ of $\operatorname{GL}(V)$ of determinant $\epsilon \in\{-1,1\}$ acts as $\epsilon$ on a 1 -space, $\delta$, and as $-\epsilon$ on a 2 -space, $\Delta$. We adopt the same terminology of center and axis (though here, of course, $\delta$ does not lie on $\Delta$ ) and write $t=t_{\delta \Delta}^{\epsilon}$. Observe that $t_{\delta \Delta}^{\epsilon}$ fixes precisely the points $\{\delta\} \cup\{\gamma: \gamma$ lies on $\Delta\}$ and the lines $\{\Delta\} \cup$ $\{\Gamma: \delta$ lies on $\Gamma\}$. If $s=t_{\gamma \Gamma}^{\epsilon^{\prime}}$, the analogue of (3) is the following:

$$
[s, t]=1 \quad \text { if and only if } \quad \gamma \in \Delta \text { and } \delta \in \Gamma \text {. }
$$

\section{Subgroups of $\mathrm{GL}\left(3, \mathbb{F}_{q}\right)$ Acting on Polytopes}

We are now ready to study flag-transitive actions of subgroups of GL( $\left.3, \mathbb{F}_{q}\right)$ on regular polytopes. Throughout this section, $\mathbb{F}_{q}$ is the finite field of $q$ elements, $V$ is a three-dimensional vector space over $\mathbb{F}_{q}$, and $G=\left\langle t_{0}, \ldots, t_{r-1}\right\rangle \leq \mathrm{GL}(V)$ is a string $C$-group. Our first result restricts the rank of $G$.

Lemma 3.1 If $G \leq \mathrm{GL}\left(3, \mathbb{F}_{q}\right)$ is a string $C$-group of rank $r$, then $r \leq 4$.

Proof Let $G=\left\langle t_{0}, \ldots, t_{r-1}\right\rangle$, let $\delta_{i}$ be the center of $t_{i}$, and let $\Delta_{i}$ be its axis $(0 \leq i \leq$ $r-1)$. We handle even and odd characteristic separately.

$\operatorname{char}\left(\mathbb{F}_{q}\right)=2$. Since $\left[t_{0}, t_{1}\right] \neq 1$, we have $\delta_{0} \notin \delta_{1}$ and $\Delta_{0} \notin \Delta_{1}$.

First suppose that $\delta_{0} \notin \Delta_{1}$ and $\delta_{1} \notin \Delta_{0}$. Let $t \in T_{\delta \Delta}$ be any involution commuting with $t_{0}$. Then either $\delta_{0}=\delta$ or $\Delta_{0}=\Delta$. In either case, it is clear that $t$ does not commute with $t_{1}$. Hence $r \leq 3$.

Next suppose that $\delta_{0} \in \Delta_{1}$ (so that $\delta_{1} \notin \Delta_{0}$ ). If $t \in T_{\delta \Delta}$ commutes with $t_{0}$ and $t_{1}$, then $\delta=\delta_{0}$ and $\Delta=\Delta_{1}$. Thus all involutions commuting with $t_{0}$ and $t_{1}$ lie in the same (abelian) transvection group. It follows that $r \leq 4$.

By symmetry, the same observations hold if $\delta_{0} \notin \Delta_{1}$ and $\delta_{1} \in \Delta_{0}$.

$\operatorname{char}\left(\mathbb{F}_{q}\right)>2$. Let $\epsilon_{i}$ denote the determinant of $t_{i}$.

First suppose that $\delta_{0} \neq \delta_{1}$ and $\Delta_{0} \neq \Delta_{1}$. Let $t=t_{\delta \Delta}^{\epsilon}$ be any involution commuting with both $t_{0}$ and $t_{1}$; then $\Delta=\left\langle\delta_{0}, \delta_{1}\right\rangle$ and $\delta=\Delta_{0} \cap \Delta_{1}$. Hence there are exactly two 
possibilities for $t$ (distinguished only by determinant), and they commute with each other. It follows that $r \leq 4$.

It remains to consider the two cases $\delta_{0}=\delta_{1}$ and $\Delta_{0}=\Delta_{1}$; by duality it suffices to consider either. Suppose that $\delta_{0}=\delta_{1}$. Since $\left[t_{0}, t_{1}\right] \neq 1$, we have $\Delta_{0} \neq \Delta_{1}$. Let $t=$ $t_{\delta \Delta}^{\epsilon}$ be any involution commuting with $t_{0}, t_{1}$, and $t_{2}$. Then $\delta_{2} \in \Delta_{0}$ (so that $\delta_{0} \neq \delta_{2}$ ), $\delta_{0}=\delta_{1}, \delta_{2} \in \Delta$ (so that $\Delta=\left\langle\delta_{0}, \delta_{2}\right\rangle$ ), and $\delta \in \Delta_{0} \cap \Delta_{1} \cap \Delta_{2}$ (so that $\delta=\Delta_{0} \cap \Delta_{1}$ lies on $\Delta_{2}$ ). Since $\delta, \delta_{1}$, and $\delta_{2}$ are not collinear, we may fix a basis $e_{1}, e_{2}, e_{3}$ of $V$ such that $\delta_{0}=\delta_{1}=\left\langle e_{1}\right\rangle, \delta_{2}=\left\langle e_{2}\right\rangle$, and $\delta=\left\langle e_{3}\right\rangle$. Relative to this basis, we have

$$
\begin{aligned}
t_{0} & =\left[\begin{array}{ccc}
\epsilon_{0} & 0 & 0 \\
0 & -\epsilon_{0} & 0 \\
0 & 0 & -\epsilon_{0}
\end{array}\right], \quad t_{1}=\left[\begin{array}{ccc}
\epsilon_{1} & 0 & 0 \\
\mu & -\epsilon_{1} & 0 \\
0 & 0 & -\epsilon_{1}
\end{array}\right] \text { and } \\
t_{2} & =\left[\begin{array}{ccc}
-\epsilon_{2} & 0 & 0 \\
0 & \epsilon_{2} & 0 \\
0 & 0 & -\epsilon_{2}
\end{array}\right]
\end{aligned}
$$

for some $\mu \neq 0$. An easy calculation now shows that $\left(t_{0} t_{1}\right)^{2}=\left(t_{2} t_{1}\right)^{2}$ is a nontrivial transvection. It follows that $\left\langle t_{0}, t_{1}\right\rangle \cap\left\langle t_{2}, t_{1}\right\rangle$ contains an element of order $p>2$, which contradicts the intersection property. Hence $r \leq 4$.

Before proceeding to our main result we record the following elementary fact.

Lemma 3.2 Let $\mathfrak{M}$ be the $\mathbb{F}_{q}$-space of $3 \times 3$ matrices with entries in $\mathbb{F}_{q}$, where $\operatorname{char}\left(\mathbb{F}_{q}\right)>2$, and let $\mathfrak{S}$ be the subspace of symmetric matrices. Then, for any involution $t \in \mathrm{GL}\left(3, \mathbb{F}_{q}\right),\left\{x \in \mathfrak{M}: x t=t^{\operatorname{tr}} x\right\} \cap \mathfrak{S}$ is a four-dimensional subspace of $\mathfrak{S}$.

Proof If $t$ has determinant $\epsilon \in\{-1,1\}$, then there exists $g \in \operatorname{GL}\left(3, \mathbb{F}_{q}\right)$ such that $\operatorname{gtg}^{-1}=t_{0}:=\operatorname{diag}(\epsilon,-\epsilon,-\epsilon)$. Evidently $\left\{x \in \mathfrak{M}: x t_{0}=t_{0}^{\mathrm{tr}} x\right\}$ is the centralizer in $\mathfrak{M}$ of $t_{0}$ and hence is five-dimensional, consisting of matrices of the form

$$
\left[\begin{array}{lll}
* & 0 & 0 \\
0 & * & * \\
0 & * & *
\end{array}\right] .
$$

It follows that $\left\{x \in \mathfrak{M}: x t_{0}=t_{0}^{\operatorname{tr}} x\right\} \cap \mathfrak{S}$ is four-dimensional. Finally observe that $x t_{0}=t_{0}^{\operatorname{tr}} x$ if and only if $\left(g^{\operatorname{tr}} x g\right) t=t^{\operatorname{tr}}\left(g^{\operatorname{tr}} x g\right)$ and that $g^{\operatorname{tr}} x g \in \mathfrak{S}$ if and only if $x \in \mathfrak{S}$. It follows that $\left\{x \in \mathfrak{M}: x t=t^{\operatorname{tr}} x\right\} \cap \mathfrak{S}$ and $\left\{x \in \mathfrak{M}: x t_{0}=t_{0}^{\operatorname{tr}} x\right\} \cap \mathfrak{S}$ have equal dimension.

Theorem 3.3 Let $V$ be an $\mathbb{F}_{q}$-space of dimension 3 , and let $G \leq \mathrm{GL}(V)$ be a string $C$-group acting irreducibly on $V$. Then char $\left(\mathbb{F}_{q}\right)>2$, and $G$ preserves a nondegenerate, symmetric bilinear form $\mathbf{f}$ on $V$.

Proof Let $G=\left\langle t_{0}, \ldots, t_{r-1}\right\rangle$ be a string C-group acting irreducibly on $V$, let $\delta_{i}$ (respectively $\Delta_{i}$ ) denote the center (respectively axis) of $t_{i}$, and, if $\operatorname{char}\left(\mathbb{F}_{q}\right)>2$, let 
$\epsilon_{i}=\operatorname{det}\left(t_{i}\right)$. Clearly $\left\langle t_{0}, t_{1}\right\rangle$ stabilizes $\Delta_{0} \cap \Delta_{1}$ and hence acts reducibly. Therefore, by Lemma 3.1, we may assume that $r \in\{3,4\}$. We again consider even and odd characteristic separately.

$\operatorname{char}\left(\mathbb{F}_{q}\right)=2$. Following the proof of Lemma 3.1, if $\delta_{0} \notin \Delta_{1}$ and $\delta_{1} \notin \Delta_{0}$, then $r=3$. Since $t_{0}$ and $t_{2}$ commute, either $\delta_{0}=\delta_{2}$ or $\Delta_{0}=\Delta_{2}$. In the former case, $G$ fixes the line $\left\langle\delta_{0}, \delta_{1}\right\rangle$; in the latter, $G$ fixes the point $\Delta_{0} \cap \Delta_{1}$.

It remains to handle the possibilities $\delta_{0} \in \Delta_{1}$ or $\delta_{1} \in \Delta_{0}$; by duality it suffices to consider just the former. Since $\delta_{2}=\delta_{0}$ or $\Delta_{2}=\Delta_{0}$, it is clear that $\left\langle t_{0}, t_{1}, t_{2}\right\rangle$ fixes $\delta_{0}$, so that $r>3$. As in the proof of Lemma 3.1, $t_{3} \in T_{\delta_{0} \Delta_{1}}$, so $G$ also fixes $\delta_{0}$.

Since $G$ is presumed to act irreducibly, it follows that $\operatorname{char}\left(\mathbb{F}_{q}\right) \neq 2$.

$\operatorname{char}\left(\mathbb{F}_{q}\right)>2$. First consider the case $r=3$.

If $t$ is an involution of $\mathrm{GL}(V)$, then $C_{\mathrm{GL}(V)}(t)$ acts by conjugation on the set of involutions of GL( $V)$ that commute with $t$. An elementary computation shows that there are two nontrivial orbits under this action, one of each determinant. Thus we may assume that $t_{0}=\operatorname{diag}\left(\epsilon_{0},-\epsilon_{0},-\epsilon_{0}\right)$ and $t_{2}=\operatorname{diag}\left(-\epsilon_{2},-\epsilon_{2}, \epsilon_{2}\right)$.

Let $t$ be any involution. By Lemma 3.2 (following the notation set up there), $\mathfrak{S}_{t}:=\left\{x \in \mathfrak{M}: x t=t^{\mathrm{tr}} x\right\} \cap \mathfrak{S}$ is a four-dimensional subspace of $\mathfrak{S}$. The space of diagonal matrices is a three-dimensional subspace of $\mathfrak{S}$, so its intersection with $\mathfrak{S}_{t}$ has dimension at least 1 . Let $0 \neq d \in \mathfrak{S}_{t_{1}}$ be diagonal. Then $d$ commutes with $t_{0}$ and $t_{2}$, so that $t_{i}^{\mathrm{tr}} d t_{i}=d$ for all $i \in\{0,1,2\}$. Hence $G=\left\langle t_{0}, t_{1}, t_{2}\right\rangle$ preserves the symmetric bilinear form on $V$ represented by the matrix $d$. If $d$ is singular, then $G$ stabilizes the radical (nullspace) of $d$ and therefore acts reducibly. If $d$ is nonsingular, then it represents a nondegenerate form $\mathbf{f}$.

Finally we consider the case $r=4$. Suppose that $\delta_{0} \neq \delta_{1}$ and $\Delta_{0} \neq \Delta_{1}$. Since $t_{3}$ commutes with both $t_{0}$ and $t_{1}$, we have $\Delta_{3}=\left\langle\delta_{0}, \delta_{1}\right\rangle$ and $\delta_{3}=\Delta_{0} \cap \Delta_{1}$. Observe that $\left\langle t_{0}, t_{1}\right\rangle$ fixes precisely two subspaces, namely $\delta_{3}$ and $\Delta_{3}$, both of which are clearly fixed by $t_{3}$. We may therefore assume that $\left\langle t_{0}, t_{1}, t_{2}\right\rangle$ acts irreducibly on $V$ and hence (by the previous case) that it preserves a nondegenerate, symmetric bilinear form on $V$. One easily checks now that $t_{3}$ also preserves that form.

It remains to deal with the cases $\delta_{0}=\delta_{1}$ and $\Delta_{0}=\Delta_{1}$. Again, by duality, it suffices to consider either case: this time we will handle the latter. Clearly both $t_{0}$ and $t_{1}$ fix $\Delta_{0}=\Delta_{1}$. However, since $\delta_{2} \in \Delta_{0}$ and $\delta_{3} \in \Delta_{1}$, we see that $t_{2}$ and $t_{3}$ also fix that line. Hence $G=\left\langle t_{0}, t_{1}, t_{2}, t_{3}\right\rangle$ acts reducibly on $V$.

\subsection{Concluding Remarks}

Since every involution of $\operatorname{PGL}\left(3, \mathbb{F}_{q}\right)$ lifts to an involution of $\operatorname{GL}\left(3, \mathbb{F}_{q}\right)$, one readily obtains a projective version of Theorem 3.3. In particular no group $G$ satisfying $\operatorname{PSL}\left(3, \mathbb{F}_{q}\right) \leq G \leq \operatorname{PGL}\left(3, \mathbb{F}_{q}\right)$ arises as the automorphisms of a regular polytope. There are, however, projective semilinear groups that admit such actions; there are various examples of regular polytopes $\mathcal{P}$ (of ranks 3 and 4) such that $\Gamma(\mathcal{P}) \cong \mathrm{P} \Gamma \mathrm{L}\left(3, \mathbb{F}_{9}\right)$.

Finally, one appealing aspect of the approach taken here (at least to the authors) is the interplay between algebra and projective geometry. The reliance on Lemma 3.2 is therefore somewhat vexing. It would be nice to have a purely geometric proof of the 
fact that a string $C$-group of rank 3 acting irreducibly on $\mathbb{F}_{q}^{3}$ preserves a nondegenerate symmetric bilinear form.

\section{References}

1. Kleidman, P.B., Liebeck, M.W.: The Subgroup Structure of the Finite Classical Groups. LMS Lecture Note Series, vol. 129. LMS, Cambridge (1990)

2. Leemans, D., Schulte, E.: Groups of type $L_{2}(q)$ acting on polytopes. Adv. Geom. 7, 529-539 (2007)

3. McMullen, P., Schulte, E.: Abstract Regular Polytopes. Encyclopedia of Mathematics and Its Applications, vol. 92. Cambridge University Press, Cambridge (2002)

4. Monson, B., Schulte, E.: Reflection groups and polytopes over finite fields, I. Adv. Appl. Math. 33, 290-317 (2004)

5. Sjerve, D., Cherkassoff, M.: On groups generated by three involutions, two of which commute. In: The Hilton Symposium 1993, Montreal, Quebec. CRM Proceedings and Lecture Notes, vol. 6, pp. 169-185. Amer. Math. Soc., Providence (1994) 